\title{
Physics of island divertors as highlighted by the example of W7-AS
}

\author{
Y. Feng, F. Sardei, P. Grigull, K. McCormick, J. Kisslinger and \\ D. Reiter $^{1}$
}

Max-Planck-Institut für Plasmaphysik, Teilinstitut Greifswald, Euratom Association,
Greifswald/Garching, Germany
${ }^{1}$ Institut für Plasmaphysik, Forschungszentrum Jülich Gmbh, Euratom Association,
Trilateral Euregio Cluster, D-52425 Jülich, Germany

E-mail: feng@ipp.mpg.de

Received 30 January 2006, accepted for publication 15 June 2006

Published 25 July 2006

Online at stacks.iop.org/NF/46/807

\begin{abstract}
Based on theoretical analysis, numerical simulations and experimental results, the paper outlines a self-consistent physics picture of the island divertor transport in W7-AS, as it emerges from the present understanding, documented over the past several years of theoretical and experimental research on the subject. Key function elements of a divertor, such as particle flux enhancement, neutral screening, impurity retention, thermal power removal via impurity line radiation and detachment, are examined for the island divertor and assessed with respect to tokamak divertors. The paper focuses on describing the global scrape-off layer (SOL) transport behaviour associated with the specific island topology and aims at illustrating the elementary differences and similarities in divertor physics between a tokamak and a typical helical device. Shown and analysed are also the correlation between the SOL and core plasma and the role of the island divertor for improving the global plasma performance. Discussion is mainly based on simple models and estimations, while three-dimensional modelling calculations serve only for control of self-consistency and for determining basic functional dependences not accessible otherwise. The island divertor physics is presented within a theoretical frame with most key issues, however, being related to experimental results.
\end{abstract}

PACS numbers: 52.55.Hc, 52.55.Rk, 52.65.-y

(Some figures in this article are in colour only in the electronic version)

\section{Introduction}

While in tokamaks divertors have been extensively investigated for several decades and the poloidal-field divertor as a successful concept has been accepted and installed in most of the existing axisymmetric devices, divertor programs for stellarators were started only recently [1-3]. An overview of the divertor activities in stellarators is given in [4]. Both theory and experiment are at the very beginning. Because of the large differences in magnetic-field topology among individual stellarators, the question as to whether there exists, like the poloidal-field divertor for tokamaks, a general divertor concept for helical devices still remains open. Nevertheless, purely from the geometry point of view, there exists at least one common feature shared by all stellarators, i.e. the existence of natural magnetic islands. Magnetic islands have a divertor potential and their feasibility and suitability for a divertor solution of plasma exhaust in low-shear stellarators has been demonstrated in W7-AS [1]. The island divertor in W7-AS significantly improved the recycling conditions, making the plasma density easily controllable even in the presence of a strong NBI-source. The divertor operation gave the discovery of a new confinement regime, the high density $\mathrm{H}(\mathrm{HDH})$-mode [5], which is characterized by high density and good energy and low impurity confinement [6]. It has been demonstrated experimentally that more than $90 \%$ of the scrape-off layer (SOL) power can be removed by impurity line radiation and the radiation layer can be stabilized outside the confinement region without a remarkable degradation of the global energy confinement. The island divertor program started in W7AS will continue in $\mathrm{W} 7-\mathrm{X}$ where the enlarged islands are even more suitable for further investigating the island divertor concept [7].

Generally, the island divertor follows the same principle as the tokamak poloidal-field divertor. The magnetic islands either have a naturally open diverting structure or are opened by intersecting target plates. The open field-lines inside the islands guide the power entering the islands across the inner separatrix towards the targets positioned at the outer separatrix. The islands form an intermediate SOL between 
the confinement core and the plasma-surface interaction region, preventing the core plasma from a direct exposure to the recycling neutrals and the sputtered impurities. On the other hand, however, large differences in the respective magnetic and divertor geometries exist between tokamaks and stellarators, including three-dimensional (3D) effects, which influence the plasma, neutral and impurity transport in the SOL. Using the W7-AS island divertor as an example, the paper shows the basic similarities and differences in SOLtransport between tokamaks and a helical device. Except for some specific effects, which are highly sensitive to the fine island structure, e.g. the location of the impurity radiation layer in detached plasmas, discussion is restricted to a physics level governed by the basic island topology. Simple models and estimations are presented, aimed mainly at illustrating and explaining the leading physics and mechanisms behind the effects observed in experiments or found by 3D simulations using the EMC3/EIRENE code $[8,9]$.

Section 2 illustrates the basic differences and similarities in divertor principle and geometry between the island divertor and the tokamak poloidal-field divertor. The characteristic transport time scales in the island divertor are compared with those in tokamaks. The island divertor configuration of W7AS is shown in section 3. In section 4, an extended twopoint model is presented in order to better understand the role of cross-field transport in the island divertor. Detailed discussion and analysis are given on some island-divertor specific effects such as the geometry-related momentum loss, absence of a high-recycling regime and drop of the upstream temperature at high densities. The EMC3/EIRENE code results are compared with measurements. Section 5 addresses the neutral-screening effect of the edge islands and the core fuelling by the recycling neutrals. Simulation results are discussed in conjunction with experimental observations. Section 6 deals with the impurity transport in the island SOL and, in particular, presents and describes the island-retention effect on intrinsically released impurities. The underlying mechanism and conditions are analysed and discussed in detail. Detachment physics is presented in section 7 where the detachment transition condition is derived from the radial energy transport including impurity radiation. Stable partial detachment, impurity radiation location, Marfe-like radiation instabilities and island-neutral screening effects in detached plasmas are briefly reviewed. More details concerning detachment stability are referred to in [10]. The major results are summarized in section 8 .

\section{Island divertor versus tokamak divertor}

\subsection{Principle and geometric aspects}

In a divertor configuration, particle and energy leaving the confinement region are guided by open field-lines to targets that are remote from the core plasma. In tokamaks this is realized by introducing additional poloidal-fields which tear the nested flux surfaces apart by forming a separatrix. The magnetic islands in low-shear stellarators provide a natural separatrix configuration without the need for any additional fields. The principles of the tokamak poloidalfield divertor and of the island divertor in stellarators are
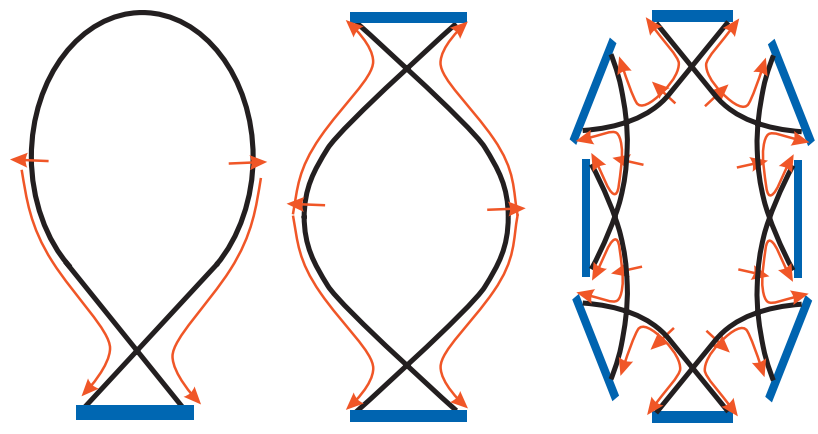

Figure 1. Sketch of divertor principle. From left to right: single and double-null poloidal-field divertors in tokamaks and the island divertor where the island number $n$ changes from device to device. For example, in W7-AS, $n$ can be changed from 8 to 10 , with $n=9$ being the standard case. In W7-X, $n$ can be chosen from 4 to 6 , with $n=5$ being standard and in LHD, $n=1$.

sketched in figure 1. Target plates are usually shaped to meet the basic geometry and symmetry of the magnetic-field configuration provided in each device. For example, the poloidal-field divertor in tokamaks is axisymmetric, while the divertor plates in stellarators have to be bent helically. In a tokamak SOL, a finite rotational transform $\iota$ leads to the termination of field-lines on targets. The target-to-target (more precisely, X-point-to-X-point) connection length can be estimated by $L_{\mathrm{c}}=2 \pi R / N \iota$ where $N$ is the null-number. In an island divertor, the rotational transform itself does not directly contribute to diverting plasma to targets. Formally, the rotational transform can be divided into two parts, i.e. $\iota=m / n+r_{\mathrm{i}} \iota^{\prime}$, where $m$ and $n$ are the respective toroidal and poloidal mode numbers, $r_{\mathrm{i}}$ the radial size of the islands and $\iota^{\prime}$ the shear at the $m / n$ resonance. The first part represents the $m / n$ resonance on which the islands reside. This part of the rotational transform does not direct field-lines from the core to the targets. The termination of field-lines on targets is due to the second, internal rotational transform $\iota_{\mathrm{i}}=r_{\mathrm{i}} \iota^{\prime}$ around the island centre (O-point). Following the connection length estimation made for tokamaks, a representative connection length in island divertors can be expressed by $L_{\mathrm{c}}=2 \pi R / N \iota_{\mathrm{i}}$, where $N=n=$ number of the islands. Thus, the connection length in an island divertor is determined by the shear. This explains why the island divertors, especially in low-shear stellarators, generally have much larger connection lengths than tokamaks, despite the multi-island structure.

In an island divertor, the target-core separation is limited by the radial extension of the islands. The radial size of the islands scales as $r_{\mathrm{i}} \propto \sqrt{R b_{m n} / n \iota^{\prime}}$, where $R$ is the major radius and $b_{m n}$ the resonant radial perturbation field normalized to the toroidal field. Low-shear stellarators generally favour largeisland formation. With additional perturbation fields, the 5/9islands in W7-AS can reach a maximum radial size of $\sim 4 \mathrm{~cm}$. The islands will become larger for a larger stellarator with a lower poloidal mode number, provided the perturbation field and the shear do not scale with the machine size within the low-shear stellarator family. For example, the 5/5-islands in W7-X can reach a radial size of $\sim 10 \mathrm{~cm}$. In the Large Helical Device (LHD), which has a large shear, an island divertor configuration is realized by choosing the lowest-n, $m / n=1 / 1$ island generated by additional perturbation fields. 


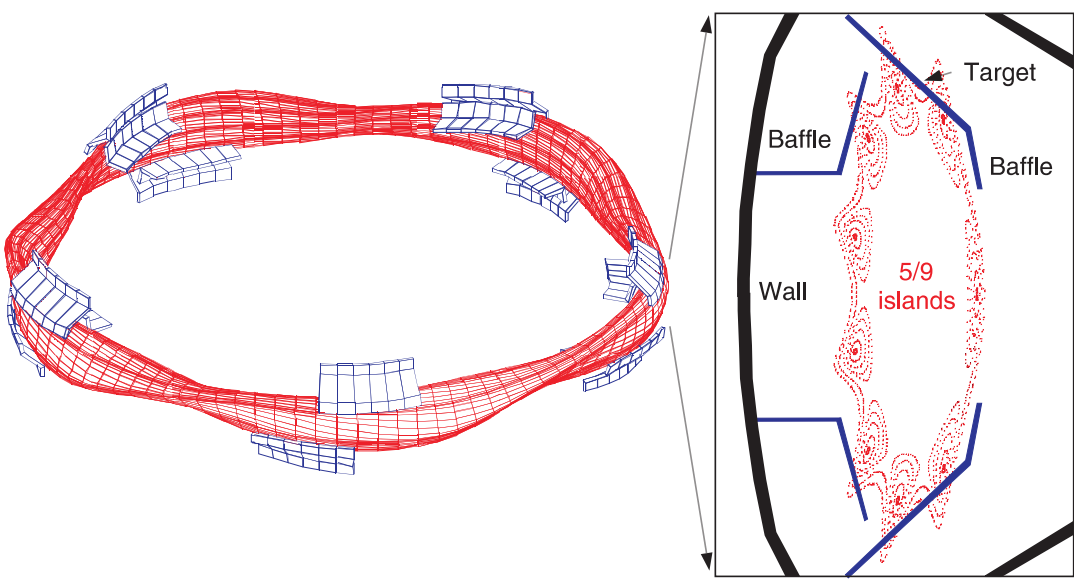

Figure 2. Standard W7-AS island-divertor configuration: nine islands are formed at the edge of a 5/9 configuration. Ten up/down symmetric divertor plates and baffles are installed on the elliptical cross-sections where the islands have the largest radial size.

\subsection{Transport characteristics}

As already mentioned, the island divertors in stellarators, compared with the poloidal-field divertor in tokamaks, usually have larger connection lengths and shorter target-tocore distances. Both enhance the perpendicular-to-parallel transport ratio in the island SOL. In the following, we compare the perpendicular-to-parallel transport ratio between tokamaks and W7-AS. For convenience, we introduce the field-line pitch $\Theta$ which, in a tokamak, is defined by $\iota a / R=B_{\mathrm{p}} / B(a=$ minor radius) and takes a typical value of 0.1 . In W7AS, the divertor-relevant field-line pitch is determined by the internal rotational transform $\iota_{\mathrm{i}}=r_{\mathrm{i}} \iota^{\prime}$, i.e. $\Theta=\iota_{\mathrm{i}} a / R$ and can only reach a value of about 0.001 which is by two orders of magnitude smaller than that in a tokamak [11]. Consider first the energy transport and assume pure heat conduction processes. The condition for the parallel heat flux to dominate over the perpendicular one in the SOL is $T_{\mathrm{e}}>$ $\left(\chi n_{\mathrm{e}} / \Theta^{2} \kappa_{\mathrm{e}}\right)^{2 / 5}$, where $\chi$ is the cross-field heat conductivity. Assuming, for example, $\chi n_{\mathrm{e}}=1 \times 10^{20} \mathrm{~m}^{-1} \mathrm{~s}^{-1}$, satisfying the above criterion requires a temperature of about $36 \mathrm{eV}$ in W7-AS and $1 \mathrm{eV}$ in a tokamak divertor, respectively. This means that, while the energy transport in a tokamak SOL is governed by the parallel heat conduction, in the island divertor the cross-field transport competes with the parallel one and even becomes dominant in low-temperature regions where $T_{\mathrm{e}}<36 \mathrm{eV}$, presenting a complex transport pattern inside the islands. For particle and momentum transport, if a classical parallel convection and an anomalous cross-field diffusion are assumed, the ratio of the parallel to the perpendicular transport time scale is given by $\tau_{\|} / \tau_{\perp}=2 D / \Delta x \Theta V_{\|}$with $\Delta x$ indicating the target-to-core distance. For $V_{\|}=8 \times 10^{4} \mathrm{~m} \mathrm{~s}^{-1}$ (sound speed of a hydrogen plasma with $T_{\mathrm{e}}=T_{\mathrm{i}}=36 \mathrm{eV}$ ) and a diffusion coefficient $D=1 \mathrm{~m}^{2} \mathrm{~s}^{-1}$, the ratio of $\tau_{\|}$to $\tau_{\perp}$ takes a value of about 0.6 in W7-AS with $\Theta=0.001$ and $\Delta x=4 \mathrm{~cm}$. This value drops down to $1 \times 10^{-3}$ when $\Theta$ increases to 0.1 and $\Delta x$ to $20 \mathrm{~cm}$, like, e.g. in a tokamak-like ASDEX-Upgrade.

\section{The W7-AS island divertor}

Figure 2 shows the standard island-divertor configuration in W7-AS. Ten identical divertor modules are installed up/down symmetrically on the elliptical planes, where the islands experience the largest radial extent, for effectively screening the recycling neutrals. The divertor plates are discontinuous for technical reasons. Each target plate toroidally extends over $18^{\circ}$ and poloidally cuts two islands in order to increase the deposition area. The baffles prevent the recycling neutrals from escaping from the divertor region towards the midplane where the magnetic islands are radially strongly compressed and optically thin for the recycling neutrals. The island size and the internal field-line pitch can be adjusted externally by ten control coils.

\section{Plasma transport}

\subsection{An extended two-point model including cross-field transport}

In order to better understand the role of cross-field transport in the island divertor, we extend the tokamak two-point model (see, for example, [12-15]) by including cross-field transport terms. For this purpose, we have to strongly simplify the island geometry. First, we do not distinguish between the direction along the island fans and the radial direction $x$ (see figure 3 ) which indicates the shortest way from core to targets.

Furthermore, we assume a spatially constant field-line pitch in the island SOL. Under this constraint, the radial one-dimensional (1D) transport equations from energy and momentum read as

$$
\begin{aligned}
& \Theta \frac{\mathrm{d}}{\mathrm{d} x}\left(-\kappa_{\mathrm{e}} T^{5 / 2} \Theta \frac{\mathrm{d} T}{\mathrm{~d} x}\right)+\frac{\mathrm{d}}{\mathrm{d} x}\left(-\chi n_{\mathrm{e}} \frac{\mathrm{d} T}{\mathrm{~d} x}\right)=0 \\
& \Theta \frac{\mathrm{d}}{\mathrm{d} x}\left(m n_{\mathrm{e}} V_{\|}^{2}+p\right)=-\frac{\mathrm{d}}{\mathrm{d} x}\left(-D \frac{\mathrm{d}}{\mathrm{d} x} m n_{\mathrm{e}} V_{\|}\right)-D \frac{m n_{\mathrm{e}} V_{\|}}{(\Delta y / 2)^{2}}
\end{aligned}
$$

with the Bohm-boundary condition at targets

$$
n_{\mathrm{ed}} c_{\mathrm{sd}} \gamma T_{\mathrm{d}}=q_{\|},
$$

where sound speed $c_{\mathrm{sd}}$ at targets is implied. The subscript ' $d$ ' in equation (3) means downstream and $\gamma$ is the sheath energy transmission factor. Volume source terms are not included in equations (1) and (2) because our attention is 


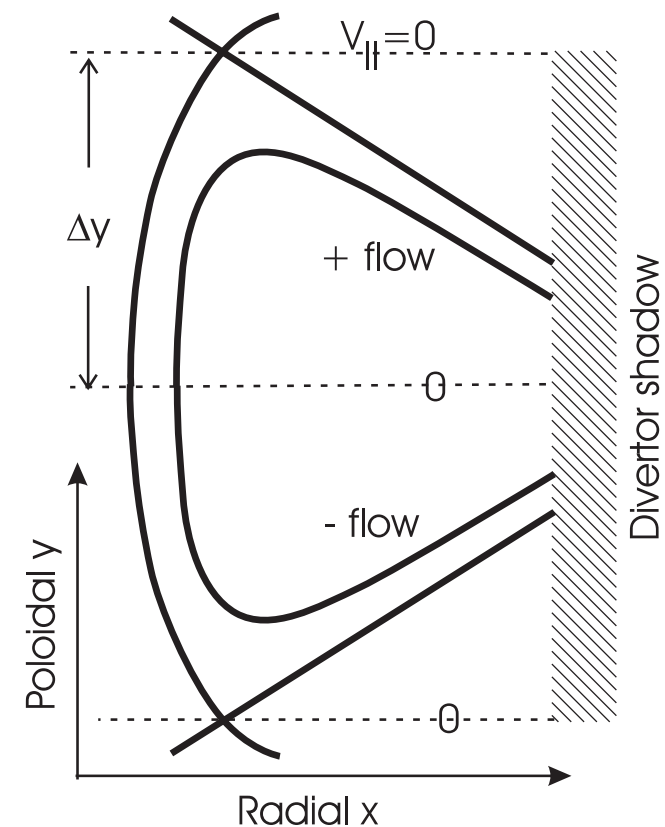

Figure 3. Simplified island geometry. Sheared positive and negative parallel flows reside on adjacent island fans. The dashed lines indicate the interfaces where plasma stagnates.

focused on transport effects. In addition, it is assumed that $T_{\mathrm{e}}=T_{\mathrm{i}} \equiv T$. The second term in equation (1) is the cross-field heat conduction contributed by both ions and electrons. The two terms on the right side of equation (2) represent the cross-field momentum transport in radial $x$ and poloidal $y$ directions as sketched in figure 3. The last term in equation (2) accounts for a pure momentum loss due to the friction between counter-flows along adjacent island fans. $\Delta y$ is the poloidal width of the flow channel limited by the interfaces between the counter-flows. On the interfaces plasma stagnates. The expression of the last term in equation (2) is obtained under the assumption of a centre-peaked flow profile. This term is important in W7-AS due to the small $\Delta y(\sim 4 \mathrm{~cm}$ on average $)$. The first term on the right side of equation (2) affects the global momentum balance through boundary conditions at upstream and downstream. This becomes clear after integrating equation (2) from upstream to downstream. Here, 'downstream' means the innermost radial position of field-lines touching the targets. Because of the discontinuous target plates, plasma ions can, through crossfield transport, leave the main flow channel and diffuse into the shadowed regions before reaching a target. A net particle flux into the shadowed region results in a convective momentum loss for the main flow channel. This process is schematically sketched in figure 4 in which a main flow channel starting from upstream terminates on the left target. In addition, part of the particles in the shadowed region flow back to the right target, causing frictional momentum loss for the ions streaming in the main channel. For simplicity, we express this momentum loss by $D m n_{\mathrm{ed}} c_{\mathrm{sd}} / \lambda_{\Gamma}$, where $\lambda_{\Gamma}$ is a characteristic decay length of the parallel flow. Assuming a zero net momentum flux across the inner separatrix, integration of equation (2) yields

$$
p_{\mathrm{u}}=2 p_{\mathrm{d}}\left(1+f_{\mathrm{m}}\right),
$$

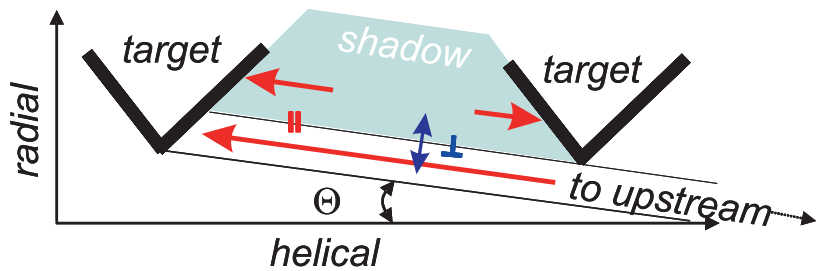

Figure 4. Schematic representation of momentum trapping in shadowed region between two local divertor plates. A main flow channel connecting to upstream position terminates on the left target. Particles diffusing into the shadowed region transport momentum away from the main channel. The backflow to the right target causes additional momentum loss for the main streaming ions by means of friction.

with

$$
f_{\mathrm{m}}=\frac{D}{2 \Theta c_{\mathrm{sd}}}\left(\frac{1}{\lambda_{\Gamma}}+\int \frac{n V_{\|} / n_{\mathrm{d}} c_{\mathrm{sd}}}{(\Delta y / 2)^{2}} \mathrm{~d} x\right) .
$$

In equation (4) $p_{\mathrm{u}}$ and $p_{\mathrm{d}}$ are the thermal pressure of ions and electrons at upstream and downstream, respectively. The momentum loss factor $f_{\mathrm{m}}$ is the integral form of the last two terms in equation (2). Replacing the density $n_{\mathrm{e}}$ in equation (1) by the averaged density between upstream and downstream, $\left(n_{\mathrm{es}}+n_{\mathrm{ed}}\right) / 2$, we have from equation (1)

$$
T_{\text {up }}^{7 / 2}=T_{\mathrm{d}}^{7 / 2}+\frac{7 q_{\|} L_{\mathrm{c}}}{2 \kappa_{\mathrm{e}}}-\frac{7 \chi\left(n_{\mathrm{es}}+n_{\mathrm{ed}}\right)}{\kappa_{\mathrm{e}} \Theta^{2}}\left(T_{\text {up }}-T_{\mathrm{d}}\right)
$$

If $\Theta \rightarrow \infty, f_{\mathrm{m}}$ and the last term in equation (6) vanish. Then, equations (3), (4) and (6) form a standard two-point model for tokamaks. For a rather small field-line pitch like that in W7-AS, however, these two terms become important. Nevertheless, some further assumptions have to be made for $f_{\mathrm{m}}$ before this simple model can be helpful for understanding the island divertor transport. In fact, the momentum loss processes are associated with the complex 3D island divertor geometry and a precise assessment is only possible using a $3 \mathrm{D}$ code. Here, we assume that both $\lambda_{\Gamma}$ and the second term in the parentheses in equation (5), i.e. the integral of the radial flow profile, remain unchanged in the island SOL. Then, $f_{\mathrm{m}}$ can be expressed as $f_{\mathrm{m}}=\alpha / T_{\mathrm{d}}^{1 / 2}$, with $\alpha$ being a parameter representing the strength of the momentum loss. Then, for a given $q_{\|}$, the downstream density and upstream and downstream temperature can be determined by equations (3)(6) using the upstream density, $n_{\mathrm{es}}$, as an independent variable. For $q_{\|}=0.5 \mathrm{MW} /\left(4 \pi^{2} a R \Theta\right)$ and $\chi=\chi_{\mathrm{e}}+\chi_{\mathrm{i}}=3 \mathrm{~m}^{2} \mathrm{~s}^{-1}$, the results are shown in figures 5 and 6 . Figure 5 shows the $n_{\text {es }}$-dependence of the downstream density for different strengths of momentum loss which is varied by changing $\alpha$. The first dashed curve shows the standard two-point model results without cross-field transport as a reference point. The sharp change in curve slope at $n_{\mathrm{es}}=1 \times 10^{13} \mathrm{~cm}^{-3}$ indicates the transition to a high recycling regime. The solid curves show the results from the extended two-point model including crossfield transport for $\alpha=0,2,5$ and 10. The small difference between the $\alpha=0$ curve and the standard case arises from the slight drop of $T_{\text {up }}$ due to the cross-field heat conduction included in the extended model, which will be discussed later in some detail. With increasing $\alpha$, the curves become flatter and flatter. For $\alpha>5, n_{\text {ed }}$ remains below $n_{\text {es }}$ and the sharp transition from low to high recycling predicted by the standard 


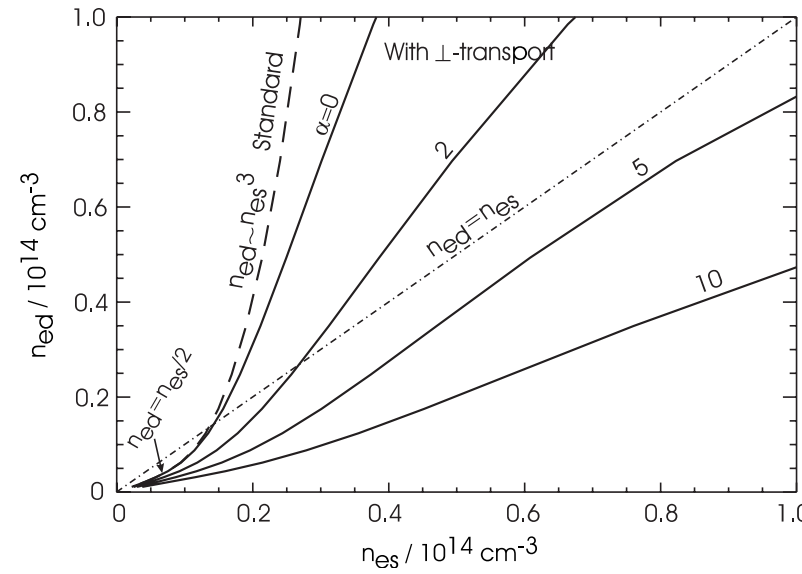

Figure 5. Downstream density versus separatrix density: comparison between the standard two-point model and the extended two-point model with different strengths of momentum loss resulting from cross-field transport.

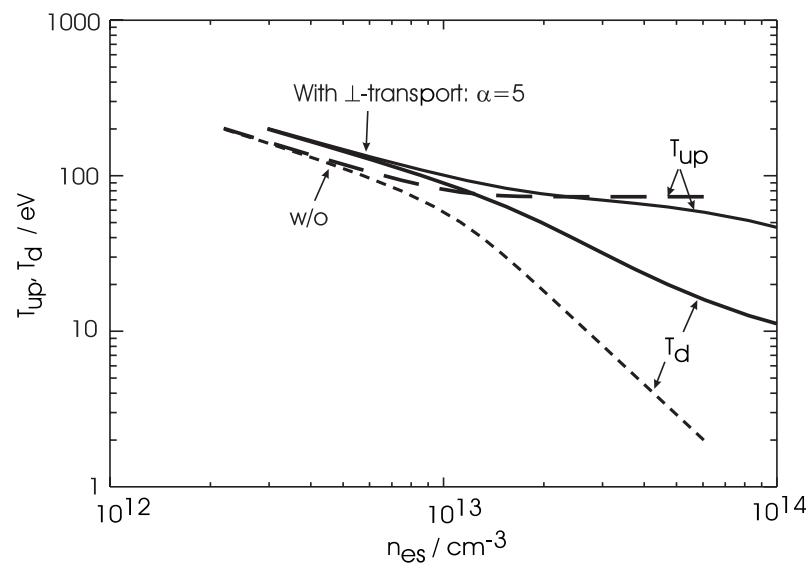

Figure 6. Upstream and downstream temperatures versus separatrix density: comparison between the standard two-point model and the extended two-point model with $\alpha=5$.

two-point model disappears. Figure 6 compares the evolution of upstream and downstream temperatures with increasing $n_{\mathrm{es}}$ between the standard case and the extended model with $\alpha=5$. Compared with the standard two-point model, the extended model shows a much more moderate change of $T_{\mathrm{d}}$ with $n_{\mathrm{es}}$ because of the weaker $n_{\text {es }}$-dependence of $n_{\text {ed }}$ just discussed before (see figure 5). This, as will be discussed in section 7.1 in detail, shifts detachment transition to extremely high $n_{\text {es }}$ values. $T_{\text {up }}$ changes its slope once decoupled from $T_{\mathrm{d}}$ as it does in the standard two-point model. However, it does not reach a plateau. Indeed, it decreases slightly due to the increased contribution from the cross-field heat conduction. At high $n_{\mathrm{es}}$, $T_{\text {up }}$ changes its slope once again. In this $n_{\mathrm{es}}$-range, the crossfield heat conduction flux tends to dominate over the parallel one, leading to a strong drop of $T_{\text {up }}$ with $n_{\text {es }}$. The significant contribution of the cross-field heat conduction to the energy transport in the islands has an important effect on impurity transport, which will be discussed in section 6 .

\section{2. $3 D$ simulations and comparison with experiments}

The plasma transport in the W7-AS island divertor has been extensively studied using the EMC3/EIRENE code on different

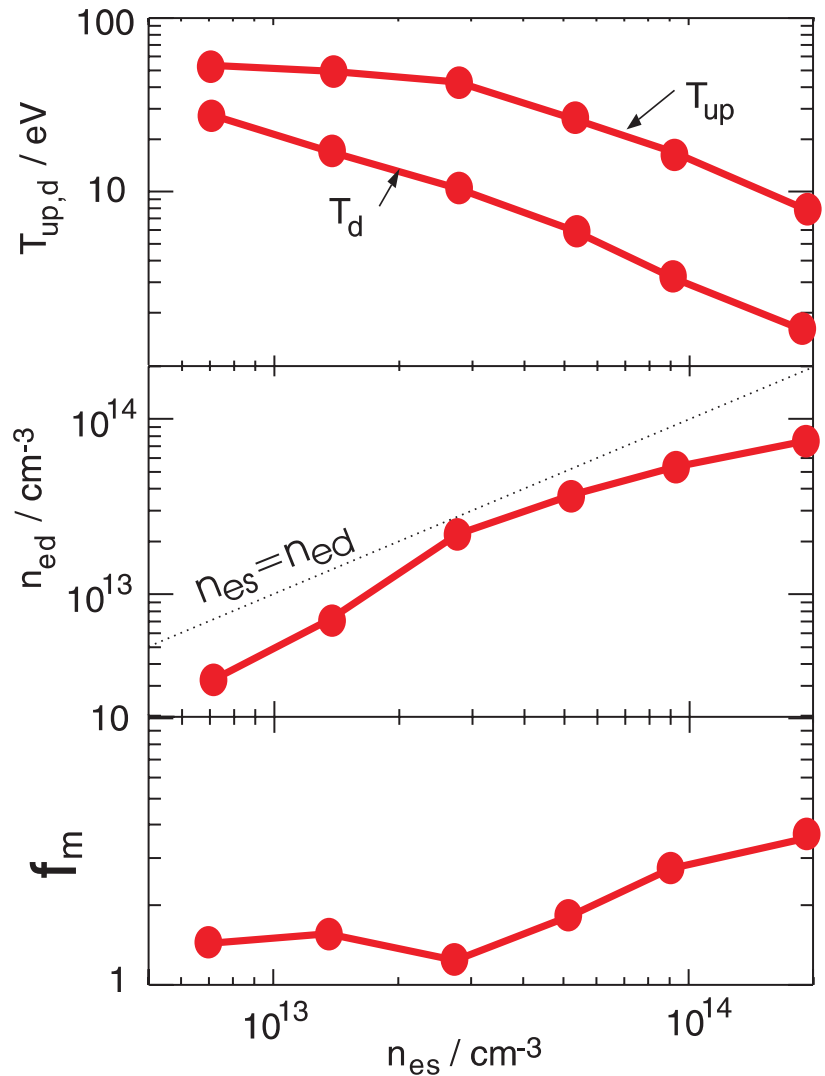

Figure 7. EMC3/EIRENE simulation results for pure hydrogen plasmas with $P_{\mathrm{SOL}}=200 \mathrm{~kW}$. From top to bottom: upstream and downstream temperatures, downstream density and momentum loss factor $f_{\mathrm{m}}$ as a function of $n_{\mathrm{es}}$.

physics and geometry levels with and without impurities for model and realistic island configurations based on the vacuum field. Input parameters like the SOL power (power entering the $\mathrm{SOL}=$ absorbed power - core-radiation) and cross-field transport coefficients, which are estimated from the probe measurements on the targets and the neutral pressure measurements in the divertor chamber, were also scanned in reasonable ranges. Details of the results depend on the individual input data set. However, some basic common features remain. Firstly, significant momentum losses already set in at low densities, high temperatures without an intensive neutral gas interaction. Secondly, as a consequence of the first point, the downstream density is almost linearly coupled with and remains smaller than the upstream density. Thirdly, significant contribution of the cross-field heat conduction to the energy transport in the islands leads usually to a drop of the upstream temperature at high density conditions.

Figure 7 shows the EMC3/EIRENE simulation results for pure hydrogen plasmas without impurities [16]. A SOL power of $200 \mathrm{~kW}$ was used. In the absence of impurity radiation, the reduced SOL power allows an examination of linear and nonlinear transport processes within a more reasonable $n_{\mathrm{es}}$-range. Shown in figure 7 are the $n_{\mathrm{es}}$-dependences of the downstream density, the upstream and downstream temperatures and the momentum loss factor $f_{\mathrm{m}}$ (see equation (4)). The 3D code confirms the basic results predicted by the extended two-point model, as highlighted above. 

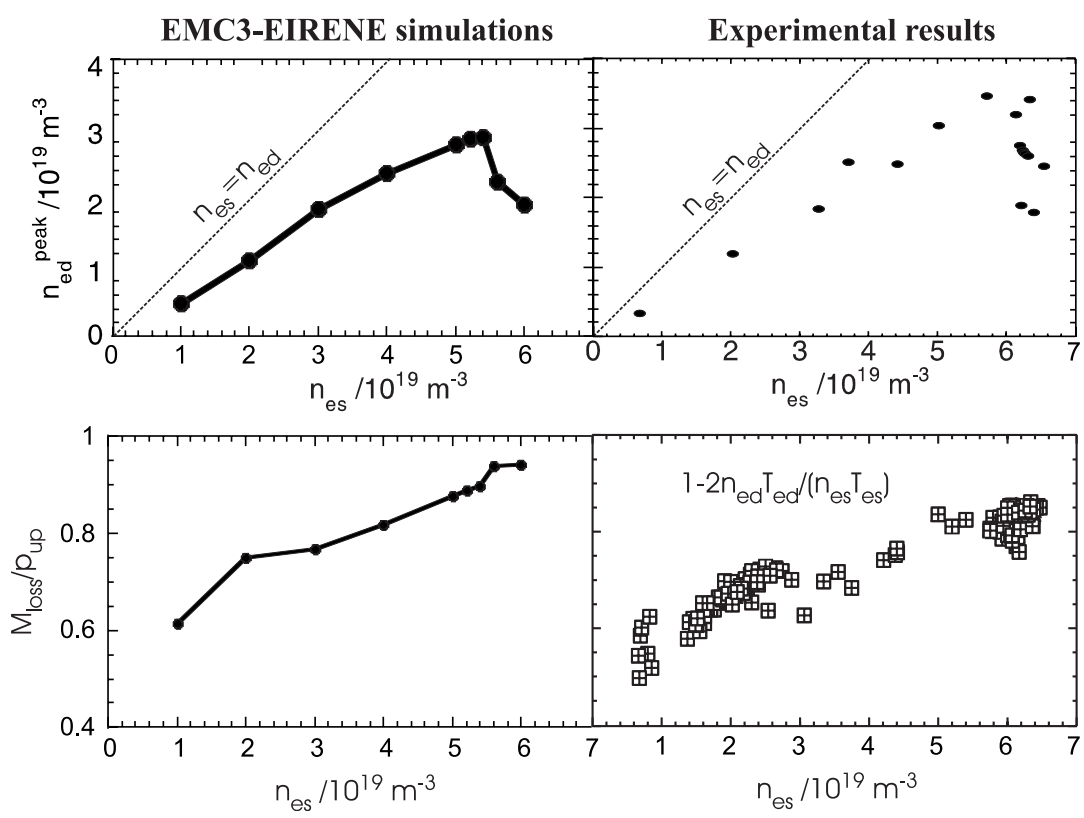

Figure 8. Absence of a high recycling regime in W7-AS. Top: correlations between downstream and upstream densities calculated by EMC3/EIRENE code (left) and measured by target Langmuir probes (right) for hydrogen plasmas heated by 2 MW NBI. Bottom: momentum loss $\left(M_{\text {loss }}=f_{\mathrm{m}} * 2 p_{\mathrm{d}}\right)$ as a function of $n_{\mathrm{es}}$ from simulations and experiments. In the experiments, an up-/downstream pressure balance analysis is only possible for electrons.

In order to make a comparison with experimental results, the intrinsically released carbon impurity has to be taken into account. Using SOL powers (0.85-1 MW) determined by experiments, an upstream-density scan was performed using the EMC3/EIRENE code [11]. The code and experimental results are compared in figure 8 . The roughly linear relation between $n_{\text {ed }}$ and $n_{\text {es }}$ found in the code simulations throughout the density range up to rollover is verified by the experiments (figure 8 top) and confirms the previous code predictions without impurities (see figure 7). Neither simulations nor experiments show any indication of transition to a high recycling regime. The pressure imbalance between upstream and downstream is shown in the bottom two pictures in figure 8 where the momentum loss $M_{\text {loss }}$ is defined by $2 p_{\mathrm{d}} f_{\mathrm{m}}$. In experiments, a pressure balance analysis is possible only for electrons. The downstream electron density and temperature are taken from the peak values of the Langmuir probes mounted on the target segments where the plasma first detaches with increasing density [17]. Both the code and experiment show significant momentum loss already at low densities. The downstream density decreases once the ionization zone moves inwards due to temperature effects (see also next section). The inward shift of the ionization front due to the reduced ionization activity at downstream causes a radial inward extension of the parallel plasma flow, leading to an enhancement of the momentum loss due to counter-flow friction. This can well be understood from equation 5 where a broadening of the radial flow profile will increase the momentum loss factor $f_{\mathrm{m}}$.

The momentum loss contribution from the chargeexchange $(\mathrm{CX})$ neutrals was examined by switching off the corresponding term in the $3 \mathrm{D}$ code. It was found that, in the attached state discussed here, a relaxed momentum balance without CX-contribution led to slight increases in the downstream density. Nevertheless, this change in $n_{\mathrm{ed}}$ remained below $10 \%$ in the given parameter range.

\section{Recycling and neutral screening}

In contrast to tokamak divertors, the open island divertor in W7-AS with relatively small islands has generally a weak neutral screening efficiency. In addition, as shown in the previous section, a strong local density rise at downstream under high-recycling conditions cannot be established in W7AS. Thus, in order to improve the island neutral screening efficiency, the upstream density has to be strongly increased to increase the overall density in the islands. A fluid approximation allows a simple estimation of the neutral penetration length [18]:

$$
\lambda_{0}=\frac{\sqrt{k T / m_{\mathrm{h}}}}{n_{\mathrm{e}} \sqrt{\langle\sigma v\rangle_{\text {ion }}\left(\langle\sigma v\rangle_{\text {ion }}+\langle\sigma v\rangle_{\mathrm{CX}}\right)}},
$$

where $\langle\sigma v\rangle_{\text {ion }}$ and $\langle\sigma v\rangle_{\mathrm{CX}}$ are ionization and charge exchange rates, respectively. In the temperature range above the ionization energy, $\lambda_{0}$ does not depend on the temperature very much and can be approximated by $\lambda_{0} \approx 1 /\left(3 \times 10^{-19} n_{\mathrm{e}}\right)$ (m) [19]. In order to get a $\lambda_{0}$ comparable with the island width in W7-AS $(\sim 4 \mathrm{~cm})$, one needs an island density of $\sim 8 \times 10^{19} \mathrm{~m}^{-3}$ ! We use this simple estimate only as a guide for discussing the basic effects related to neutrals, and leave a stricter treatment to the EIRENE code. Increasing the density in the islands decreases the neutral penetration length as long as the island temperature is kept higher than the ionization energy. Once the temperature drops below this point, the ionization rate will be strongly reduced, which cannot be compensated by the density effect, leading to a deeper penetration of neutrals. Thus, $\lambda_{0}$ reaches a minimum before the ionization zone lifts from the targets.

Figure 9 shows the dependences of the total recycling flux $\Gamma_{\text {recy }}$ and the neutral flux penetrating into the core (core-fuelling rate) $\Gamma_{\text {core }}$ on the separatrix density $n_{\mathrm{es}}$ [20]. 




Figure 9. $n_{\mathrm{es}}$-dependences of the total recycling flux and the recycling flux penetrating into the core.

The results are obtained by EMC3/EIRENE simulations for hydrogen plasmas with the standard divertor configuration based on the vacuum field. Further conditions are $P_{\mathrm{SOL}}=$ 1.2 MW, $2 \%$ carbon sputtering, $D=0.5 \mathrm{~m}^{2} \mathrm{~s}^{-1}$ for both carbon and hydrogen and $\chi_{\mathrm{i}}=\chi_{\mathrm{e}}=3 \mathrm{D}$. In the calculations, $n_{\mathrm{es}}$ is increased until the plasma approaches detachment. Detailed studies on the neutral transport behaviour in detached plasmas can be found in [10]. Here, we restrict our attention to the attached case. $\Gamma_{\text {core }}$ is normalized to the NBI-source in order to show the relative importance of the core fuelling by the recycling neutrals. In the low $n_{\mathrm{es}}$-range $\left(n_{\mathrm{es}}<2 \times 10^{13} \mathrm{~cm}^{-3}\right)$, the islands are transparent to the recycling neutrals (large $\lambda_{0}$ ) so that $\Gamma_{\text {core }}$ increases with the $n_{\mathrm{es}}$ as a consequence of the rising total recycling flux $\Gamma_{\text {recy. }}$. Increasing $n_{\text {es }}$ decreases $\lambda_{0}$ and leads to a movement of the ionization front towards the targets. Around $n_{\mathrm{es}}=2 \times 10^{13} \mathrm{~cm}^{-3}$, the island screening effect compensates the source (increase of $\Gamma_{\text {recy }}$ ) effect and stops the growth of $\Gamma_{\text {core }}$. After this point, $\Gamma_{\text {core }}$ drops slightly due to the gradually-improved island screening efficiency until the downstream temperature drops below the ionization energy of the recycling neutral gas. Then, the strongly-reduced ionization activity makes the islands transparent again for the recycling neutrals. In this case, $\Gamma_{\text {core }}$ increases quickly with $n_{\mathrm{es}}$. Impurity radiation strengthens this process.

The recycling neutrals provide the main fuelling source for the core (see figure 9), which should be reflected by the core density. Figure 10 shows the experimentally measured dependence of the line-averaged density on the separatrix density. Comparing with the $\Gamma_{\text {core }}$-curve shown in figure 9 , one sees a clear correlation between the calculated core-fuelling rate and the measured line-averaged density. Following the rollover of $\Gamma_{\text {core }}$, the line-averaged density flattens in the $n_{\mathrm{es}}$-range from 2 to $6 \times 10^{13} \mathrm{~cm}^{-3}$ and recovers when the islands become transparent again for the recycling neutrals. In addition to the source effect, a change in core density profile due to redistribution of ionization sources or change in core transport can also modify the line-averaged density. Indeed, the plasma changes its confinement state in this $n_{\mathrm{es}}$-range and jumps from normal confinement (NC) $\left(n_{\mathrm{es}}<2 \times 10^{13} \mathrm{~cm}^{-3}\right.$

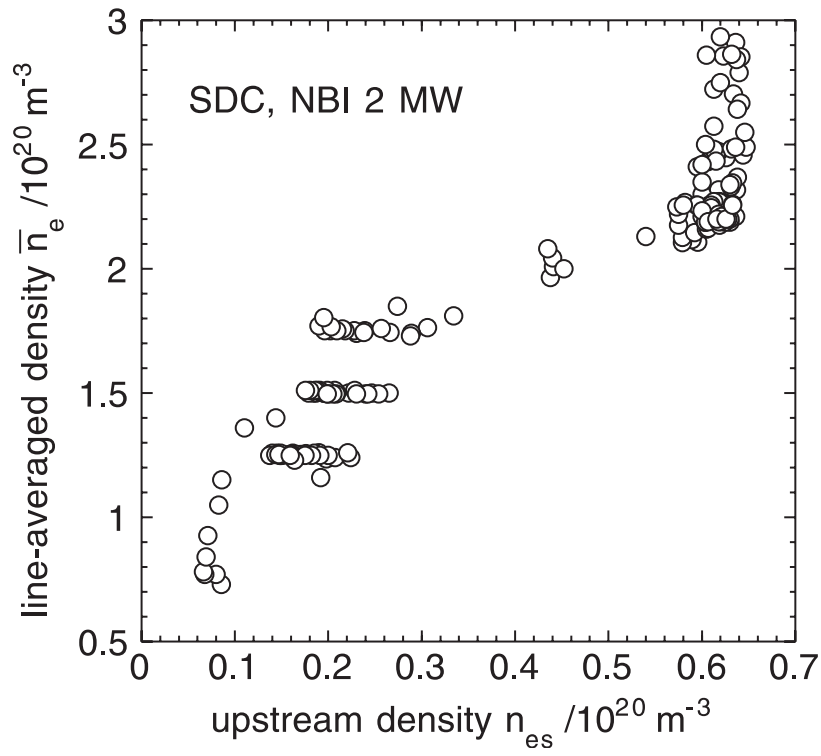

Figure 10. Correlation between the line-averaged density and the separatrix density observed in experiments for hydrogen plasmas heated by $2 \mathrm{MW}$ NBI. SDC: standard island divertor configuration.

in figure 10) to high density H-mode (HDH) $\left(n_{\mathrm{es}}>6 \times\right.$ $10^{13} \mathrm{~cm}^{-3}$ ) [21]. In contrast to the NC-plasmas, the HDHmode has an improved energy confinement and exhibits broader density profiles. An improved particle confinement, however, would lead, for a given particle-source distribution, to a steepening of the line-averaged density with respect to $n_{\mathrm{es}}$, which is obviously in contradiction to the experimental results shown in figure 10. A consistent picture could be recovered by assuming that the transport effect is overcompensated by the outward shift of the source distribution due to the broad density profile in the $\mathrm{HDH}$-mode. However, a quantitative assessment would need a detailed core particle transport analysis, which is beyond the scope of the presented paper.

In any case, the essential message here is that the island neutral screening effect can well explain the jump of the separatrix density from $\mathrm{NC}$ to $\mathrm{HDH}$ observed in experiments. In W7-AS, additional gas puffing is applied for increasing the plasma density. Gas puffing provides a primary particle source in the island SOL. The particle source increases the island density and thereby enhances the recycling process. The core plasma is indirectly fed by the recycling neutrals. A successful core density control through gas puffing needs a monotone, positive dependence of the core-fuelling rate $\Gamma_{\text {core }}$ on $n_{\text {es }}$. This is true only in low and high $n_{\mathrm{es}}$-range, as shown in figure 9. In between, there exists an $n_{\mathrm{es}}$-region where gas puffing does not feed the core plasma due to the island screening effect. In order to further increase the core density, the island neutral screening effect has to be first overcome. This can be realized by increasing $n_{\mathrm{es}}$ to cool down the islands. This explains why strong gas puffing in experiments is necessary in order to access the HDH-plasmas.

\section{Impurity retention in the islands}

Impurity transport in the SOL provides the necessary boundary conditions for the core through which it enters the impurity 
global confinement process. EMC3/EIRENE code simulations show that the edge magnetic islands in W7-AS, under highdensity, low-temperature (HDH) conditions, have a strong retention effect on the target-released carbon impurity [20].

The balance between thermal force and friction determines the parallel flow direction of impurities and thereby influences the impurity distribution in the SOL:

$$
\begin{aligned}
V_{Z \|} & =V_{i \|}+\frac{\tau_{Z i} Z^{2}}{m_{Z}}\left(0.71 \nabla_{\|} T_{\mathrm{e}}+2.2 \nabla_{\|} T_{\mathrm{i}}\right)+\frac{\tau_{Z i} Z e}{m_{Z}} E_{\|} \\
& -\frac{\tau_{Z i}}{n_{Z} m_{Z}} \nabla_{\|} T_{\mathrm{i}} n_{Z} .
\end{aligned}
$$

The first term on the right side of equation (8) is the parallel flow velocity of the background ions, the second one denotes thermal forces from electrons and ions, the third one is the parallel E-field determined by electron momentum balance and the last term is the pressure gradient of the impurities $\left(T_{\mathrm{Z}}=T_{\mathrm{i}}\right)$. While a thermal force resulting from a parallel temperature gradient tends to draw impurities upwards towards the high temperature region (upstream), a frictional plasma flow drives the impurities back to the target (downstream). Here, we ignore the flow reversal effect discussed in tokamaks. In the following, we make a simple analysis on the force balance and impurity transport processes before showing the 3D code results. The dominating thermal force comes from ions due to the weak classical conductivity with respect to electrons. The impurity pressure gradient force can be split into two parts, i.e. $\nabla_{\|} T_{\mathrm{i}}$ and $\nabla_{\|} n_{\mathrm{z}}$. The former can be neglected in comparison with the ion thermal force, especially for $Z>1$. The density gradient part represents a parallel diffusion. It can be shown that this parallel diffusive process has a much weaker effect on the impurity density distribution than the cross-field diffusion due to the small field pitch in W7-AS. Therefore, we ignore the pressure gradient term completely. Keeping only the friction and the ion thermal-force terms, the impurity flow velocity becomes independent of $Z$. Thus, the impurity continuity can be integrated over all the ionization stages. Following the simplified island geometry shown in figure 3 , the $1 \mathrm{D}$ continuity for a source-free case reads as

$$
\frac{\mathrm{d}}{\mathrm{d} x}\left(\Theta V_{Z \|} n_{\mathrm{I}}-D \frac{\mathrm{d} n_{\mathrm{I}}}{\mathrm{d} x}\right)=0,
$$

where $n_{\mathrm{I}}$ is the impurity density (sum over all $Z$ with $Z>0$ ) and $D$ is the perpendicular diffusivity. For intrinsicallyreleased impurities like the target-released carbon, the solution of the above equation is straightforward:

$$
n_{\mathrm{Is}}=n_{\mathrm{Id}} \exp \left(-\int \frac{\Theta V_{Z \|}}{D} \mathrm{~d} x\right)
$$

where $n_{\text {Is }}$ and $n_{\text {Id }}$ are the impurity density at upstream and downstream, respectively. The integration is performed from upstream to downstream. It is evident that a large, positive (outward directed) impurity flow velocity reduces the impurity density at the separatrix which is determined by the balance between the friction and the ion thermal force. As the ratio of friction to thermal force is determined by the ratio of the ion classical convective to conductive heat flux [22], both increasing the plasma flow and reducing the conductive heat flux favour the impurity retention in the islands. Based on the simplified island geometry, the radial energy transport for ions can be written as

$$
5 / 2 \Theta n T_{\mathrm{i}} V_{i \|}-\kappa_{\mathrm{i}} T_{\mathrm{i}}^{5 / 2} \Theta^{2} \frac{\mathrm{d} T_{\mathrm{i}}}{\mathrm{d} x}-\chi_{\mathrm{i}} n \frac{\mathrm{d} T_{\mathrm{i}}}{\mathrm{d} x}=q_{\mathrm{i}},
$$

where $q_{\mathrm{i}}$ is the ion energy flux coming from the core. The cross-field heat conduction builds a bypass through which the ion classical heat conductive flux can, under high-density, low-temperature conditions, be strongly reduced, leading to a significant reduction of the related thermal force. The crossfield heat conduction becomes even more dominant under the condition

$$
n / T_{\mathrm{i}}^{5 / 2}>k_{\mathrm{i}} \Theta^{2} / \chi_{\mathrm{i}}
$$

Because of the rather small $\Theta\left(\sim 10^{-3}\right)$ in W7-AS, it is possible to reach the above condition. For example, assuming that $\chi_{\mathrm{i}}=2 \mathrm{~m}^{2} \mathrm{~s}^{-1}$ and $n=5 \times 10^{19} \mathrm{~m}^{-3}$, satisfying the above condition requires $T_{\mathrm{i}}<\sim 120 \mathrm{eV}$. There are no ion temperature measurements for the islands in W7-AS. Instead, the upstream electron temperature can be measured by a Thomson scattering system. For the attached hydrogen plasmas with $2 \mathrm{MW}$ NBI, the measured upstream $T_{\mathrm{e}}$ takes a typical value of about $100 \mathrm{eV}$ [23]. For $n_{\mathrm{es}}=4 \times 10^{19} \mathrm{~m}^{-3}$, the EMC3 code, with the input parameters given in section 5 , provides an upstream $T_{\mathrm{e}}$ of about $90 \mathrm{eV}$ and $T_{\mathrm{i}}$ of $100 \mathrm{eV}$, showing no significant difference between $T_{\mathrm{e}}$ and $T_{\mathrm{i}}$, as usually expected for high density conditions. Once condition (12) is established at upstream, the cross-field heat conduction will dominate the ion energy transport throughout the islands. Then, the classical conductive heat flux is strongly reduced and thereby the related thermal force.

Figure 11 shows the balance between ion thermal force and friction for a low and an enhanced recycling case with $n_{\mathrm{es}}=1.5$ and $5 \times 10^{13} \mathrm{~cm}^{-3}$, respectively, resulting from the same 3D simulation series as shown in section 5 . At lower $n_{\text {es }}$, the thermal force dominates over the friction and exhibits a phase distribution among the island fans which results in an inward flow of impurities, as indicated by the arrows. At higher $n_{\mathrm{es}}$, the friction prevails against the thermal forces and the phase distribution of the net-force is completely reversed. The impurity flow is changed from inward- to outward-directed in almost the whole island SOL. This 'flow reversal' makes a strong impact on the impurity distributions. Figure 12 shows the corresponding carbon density (sum over all ionization stages) distributions which are normalized to the total carbon production rate in order to isolate the transport effects from the production processes. The dominating thermal force in the lower recycling case draws the carbon towards the separatrix, leading to an impurity accumulation there, whereas the plasma flow, once exceeding the thermal force, drives the impurities outwards and keeps them around the targets. This 'flow reversal' of impurity reduces the carbon density at separatrix by more than one order of magnitude. Figure 13 shows the transition process from thermal-forcedominated to friction-dominated impurity transport. One sees a sharp decrease in the carbon density at the separatrix when the friction becomes dominating over the thermal force in the $n_{\text {es }}$-range $2-3 \times 10^{13} \mathrm{~cm}^{-3}$

As discussed above, the effective reduction of the parallel conductive heat flux is associated with the small internal fieldline pitch of the islands, which is obviously stellarator-specific. 
a)
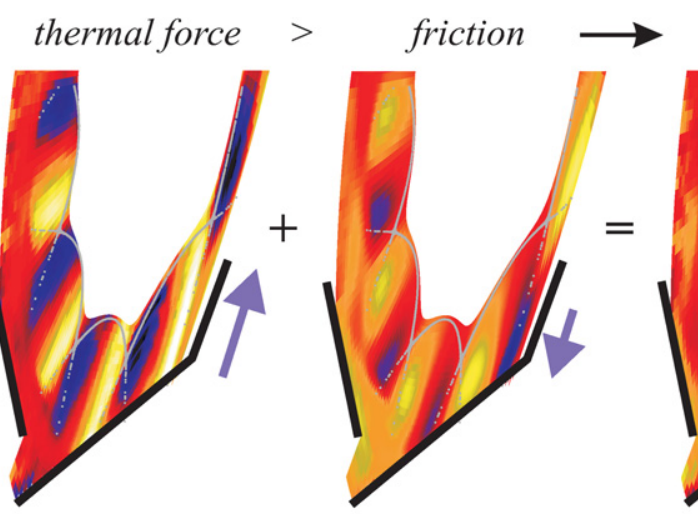

inward flow

thermal force $<$

friction

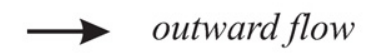

b)



Figure 11. Force balance analysis between ion thermal force and friction for $(a) n_{\mathrm{es}}=1.5 \times 10^{13}$ and $(b) 5 \times 10^{13} \mathrm{~cm}^{-3}$. The phase distribution of the frictional flow results in an outward-directed convection for impurities, as indicated by the arrows. In contrast, the thermal force exhibits an opposite phase distribution, drawing impurities towards separatrix. In the low density case $(a)$, thermal force dominates over friction, resulting in an inward flow of impurities. The flow is completely reversed for the friction-dominated case $(b)$.

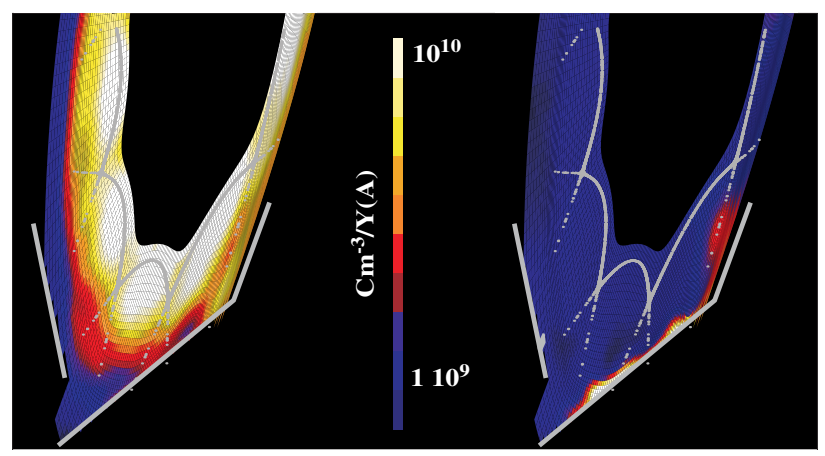

Figure 12. Distributions of carbon density normalized to carbon production rate for a thermal-force-dominated (left) and a friction-dominated case (right). The thermal force draws carbon inwards, leading to a density accumulation at the separatrix, while the friction force drags the carbon back to the target.

On the other hand, a small field-line pitch increases also the perpendicular-to-parallel transport ratio for impurities and the island impurity flushing effect will, of course, vanish when $\Theta \rightarrow 0$, which can be seen in equation (10). In reality, as already estimated in section 2.2 , the parallel convection and the cross-field diffusion play a comparable role for particle transport in the W7-AS islands. This obviously holds for impurities as well, as $V_{Z \|} \approx V_{i \|}$ for friction-dominated impurity transport, see equation (8). Furthermore, the parallel particle convection is not as sensitive to the plasma temperature as the parallel heat conduction. A larger field-line pitch



Figure 13. Carbon separatrix density as a function of $n_{\mathrm{es}}$. The carbon density at the separatrix drops quickly once the friction becomes dominant.

will increase the parallel-to-perpendicular transport ratio and thereby improve the impurity retention effect of the edge islands. This, however, would shift the transition condition to even higher densities and lower temperatures.

Besides the transport effects, impurity production, which is not addressed in this paper, is essential to finally determine 
the absolute impurity density. If the total carbon production rate increases linearly with $\Gamma_{\text {recy }}$, the impurity screening effect resulting from transport, i.e. the reduction of carbon density at the separatrix, would be, to a large extent, compensated by the increased carbon production rate. This assumption for carbon is reasonable because of chemical sputtering effects. In addition, EMC3 detachment-related simulations [10] based on a linear ansatz for carbon release agree well with experiments (see also next section). Nevertheless, light impurities like carbon do not account for a core radiation. A core-peaked radiation profile as found in the NC-plasmas should be associated with heavier impurity species. For heavy impurities like iron, a cold island plasma is essential for reducing the sputtering yield, which can be realized under enhanced recycling conditions. A quantitative assessment on the content of heavier impurities in the island SOL including self-consistently impurity production processes is beyond the scope of this paper.

\section{Detachment}

The detachment regime $[24,25]$ is attractive in its large radiation fraction which is, however, only achievable under a degraded recycling condition, meaning that the particle flux to the targets has to be reduced [26]. This requires a pressure drop along the field-lines which, in tokamaks, is realized by means of ion-neutral friction [14]. As already shown in section 4 , in W7-AS, the friction between counter-flows can cause a significant pressure drop without an intensive ion-neutral interaction. This has been evidenced in figure 8 where both the simulations and the experiments show that $n_{\text {ed }}$ (also the particle flux) drops already before detachment transition. On the other hand, in a detached plasma the power balance in the SOL is governed by impurity radiation. An intensive impurity radiation causes usually plasma condensation (strongly-localized cold, dense plasma) [27], forming highly-concentrated radiation bands/layers known as MARFEs [28] from tokamaks. The tokamakMARFE, which is poloidally asymmetric, preserves, however, the tokamak axisymmetry. This section shows how the detached plasma behaves in a non-axisymmetric helical device.

\subsection{Detachment transition}

In W7-AS carbon is the main impurity species because the targets are made of graphite. Carbon radiates intensively only when $T_{\mathrm{e}}$ approaches about $10 \mathrm{eV}$. Because of the absence of a high-recycling regime in W7-AS, the downstream temperature drops very slowly with increasing $n_{\mathrm{es}}$ so that a rather high $n_{\mathrm{es}}$ is needed in order to reach the carbon radiation temperature, which has been shown both by the extended two-point model and by the EMC3/EIRENE code (see section 4). Furthermore, carbon concentration and plasma density should be high enough for sufficient radiation to drive energy detachment. In the following, before showing the 3D code simulation results, we first analyse how and what plasma parameters and physics quantities enter the condition for detachment transition. The transition condition is determined by the balance between heat transport and radiation. We begin with the $1 \mathrm{D}$ radial energy transport described by equation (1) in section 4 and, now, take the carbon radiation into account:

$$
\mathrm{d} q / \mathrm{d} x=-C n^{2} L(T),
$$

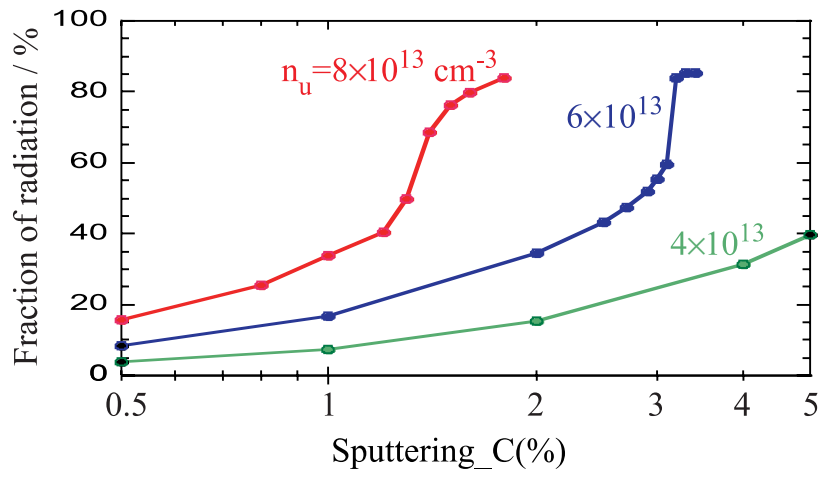

Figure 14. EMC3 predicts high upstream density conditions for detachment transition in W7-AS and a sharp increase in carbon radiation at the transition to detachment.

where $q$ is the radial heat flux, $C$ is the carbon concentration and $L$ is the cooling rate as a function of $T\left(T \equiv T_{\mathrm{e}}=\right.$ $\left.T_{\mathrm{i}}\right)$. Around the carbon radiation temperature $(\sim 10 \mathrm{eV})$, the classical parallel electron heat conduction plays a negligible role in the radial energy transport in comparison with the perpendicular one, which has been clarified in sections 3 and 4 . Using $q=\chi n \mathrm{~d} T / \mathrm{d} x$, integrating equation (13) in temperature space yields

$$
q_{\mathrm{u}}^{2}-q_{\mathrm{d}}^{2}=\int_{T_{\mathrm{d}}}^{T_{\mathrm{u}}} 2 C \chi n^{3} L(T) \mathrm{d} T,
$$

where $T_{\mathrm{u}}$ and $T_{\mathrm{d}}$ are the respective upstream and downstream temperatures and $q_{\mathrm{u}}$ and $q_{\mathrm{d}}$ are the heat fluxes. Detachment occurs when $T_{\mathrm{d}} \approx 0$ and $q_{\mathrm{d}} \approx 0$, meaning that all the power entering the island SOL is radiated by carbon impurity. We assume that the characteristic variation lengths of $C$ and $n$ in the islands are larger than the radial width of the radiation layer. In this case, we approximate $L(T)$ by $\delta$-function in $T$-space. Then, we obtain from equation (14):

$$
n=\text { const. }\left(\frac{q_{\mathrm{u}}^{2}}{C \chi}\right)^{1 / 3},
$$

with const. representing the integration constant of the cooling rate. Equation (15) indicates that, for a given $q_{\mathrm{u}}$, the carbon concentration and the cross-field heat conductivity play a less important role than the plasma density in triggering detachment.

The condition for detachment transition was examined in upstream density and carbon sputtering coefficient space using the EMC3/EIRENE code. A fixed SOL power of $1 \mathrm{MW}$ was used and, furthermore, $D=0.5 \mathrm{~m}^{2} \mathrm{~s}^{-1}$ and $\chi_{\mathrm{e}}=\chi_{\mathrm{i}}=3 \mathrm{D}$ were assumed. In the low-temperature range around $10 \mathrm{eV}$, chemical sputtering is considered to be the dominating carbon production process. In simulations, carbon is generated randomly according to the particle (background ion) outflow distribution on the targets. The absolute yield is controlled by a sputtering coefficient which is varied from 0.5 to $5 \%$. The results are shown in figure 14 . The transition to detachment can be identified by the abrupt increase in radiation for the two high density cases with $n_{\mathrm{es}}\left(n_{\mathrm{u}}\right.$ in figure 14) $=6 \times 10^{13}$ and $8 \times 10^{13} \mathrm{~cm}^{-3}$. For the $n_{\mathrm{es}}=8 \times 10^{13} \mathrm{~cm}^{-3}$ case, detachment occurs at a sputtering coefficient of $\sim 1.3 \%$ which has to be increased by more than a factor 2 up to $3.1 \%$ when $n_{\mathrm{es}}$ is 


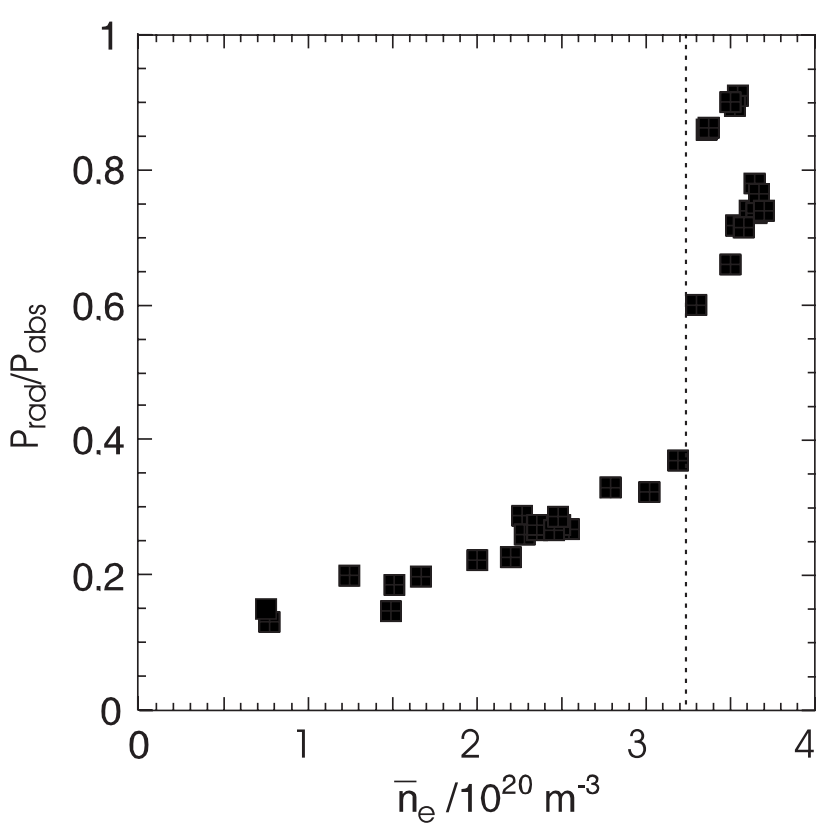

Figure 15. Bolometer measurements of total radiation as a function of line-averaged plasma density for quasi-stationary hydrogen discharges heated by $2 \mathrm{MW}$ NBI. Sharp transition to detachment is observed at a line-averaged density of $3.2 \times 10^{14} \mathrm{~cm}^{-3}$

$\left(n_{\mathrm{es}}=6 \times 10^{13} \mathrm{~cm}^{-3}\right)$ and indicated by the dashed line.

decreased by $25 \%$ down to $6 \times 10^{13} \mathrm{~cm}^{-3}$. These 3D results confirm the high-density condition for achieving detachment in W7-AS expected by the extended two-point model and are consistent with the parameter sensitivity analysis given by equation (15) if the carbon concentration $C$ in equation (15) is replaced by the sputtering coefficient.

In experiments, an abrupt detachment transition for hydrogen plasmas heated by $2 \mathrm{MW}$ NBI (absorbed $=1.4 \mathrm{MW}$ and $P_{\mathrm{SOL}}=0.8 \mathrm{MW}$ ) was found at a line-averaged density of $3.2 \times 10^{14} \mathrm{~cm}^{-3}$ and $n_{\mathrm{es}}=6 \times 10^{13} \mathrm{~cm}^{-3}$ [29], confirming the code predictions. These experimental results are shown in figure 15 .

The abrupt change in radiation level at detachment transition is associated with a thermal instability due to the twobranch characteristic of the carbon cooling rate function. This can be qualitatively understood by analysing the power balance at detachment transition. In equation (14), three parameters can be varied independently to trigger detachment, namely the impurity concentration $\boldsymbol{C}$, the plasma density $n$ and the SOL power flux $q_{\mathrm{u}}$. Each one can be used as a detachmenttrigger and the choice of the trigger does not affect the issues of the following stability analysis. For simplicity, we change $q_{\mathrm{u}}$ while fixing $C$ and $n$. The response of $T_{\mathrm{d}}$ to a change of $q_{\mathrm{u}}$ is determined by the derivative of $T_{\mathrm{d}}$ with respect to $q_{\mathrm{u}}$ :

$$
\frac{\partial T_{\mathrm{d}}}{\partial q_{\mathrm{u}}}=-\frac{q_{\mathrm{u}} / C \chi n^{3}}{L\left(T_{\mathrm{d}}\right)-F\left(T_{\mathrm{d}}\right)},
$$

where the Bohm boundary condition $q_{\mathrm{d}}=\Theta n c_{\mathrm{sd}} \gamma T_{\mathrm{d}}$ is assumed and $F\left(T_{\mathrm{d}}\right)=3 q_{\mathrm{d}}^{2} /\left(2 C \chi n^{3} T_{\mathrm{d}}\right)$. Here, energy losses due to plasma-neutral interaction are ignored. The sign and magnitude of $\partial T_{\mathrm{d}} / \partial q_{\mathrm{u}}$ depends on the relative magnitude of $F$ and $L$, which is a function of $T_{\mathrm{d}}$. A stable solution exists only when $\partial T_{\mathrm{d}} / \partial q_{\mathrm{u}}>0$ i.e. $F\left(T_{\mathrm{d}}\right)>L\left(T_{\mathrm{d}}\right)$. While $F\left(T_{\mathrm{d}}\right)\left(\propto T_{\mathrm{d}}^{2}\right)$ is a monotone function of $T_{\mathrm{d}}, L\left(T_{\mathrm{d}}\right)$ for carbon has a maximum around $T_{\mathrm{d}}=10 \mathrm{eV}$ and decreases rapidly when $T_{\mathrm{d}}$ deviates from the maximum radiation temperature. It can be shown that, in the vicinity of the maximum radiation temperature, $L$ may exceed $F$, forming an unstable range [11]. Outside this range, $F$ is larger than $L$ and stable solutions to equation (3) exist in both lower and higher temperature ranges. The unstable range is not accessible so that the plasma jumps from a high- to low-temperature solution at detachment transition, which has been shown both in the experiments and simulations (see the $n_{\mathrm{u}}=6 \times 10^{13}$ curve in figure 14). For a given $q_{\mathrm{u}}$, the product of $C$ and $n^{3}$ at detachment transition is fixed by equation (15). Therefore, for any given $T_{\mathrm{d}}, F$ scales with $n^{2}$ because of the linear dependence of $q_{\mathrm{d}}$ on $n$, meaning that shifting detachment transition to a higher density by reducing $C$ leads to an overall increase in the function $F\left(T_{\mathrm{d}}\right)$. In this case, the unstable region with $F<L$ shrinks and eventually disappears. Even so, the fact that the magnitude of $\partial T_{\mathrm{d}} / \partial q_{\mathrm{u}}$ reaches a maximum at the maximum radiation temperature means always a drastic change of plasma state (see the highest density curve in figure 14). More details concerning the thermal instability at detachment transition can be found in [11].

\subsection{Stable detachment}

After detachment transition, the radiation zone lifts from the targets and the radiation distribution in the islands is determined by the local balance between energy transport and radiation. A detached plasma is stable only when such a local power balance can be established in the island SOL. In fact, it was experimentally found that stabilizing a detached plasma in W7-AS needs sufficiently large islands with sufficiently large field-line pitch. For small islands with small field-line pitch, plasmas after detachment transition fall immediately into an unstable state. These experimental findings have motivated a detailed numerical study [10] to understand how the island geometry can affect the stability of a detached plasma. EMC3/EIRENE simulations show that the location of the carbon radiation zone at detachment is influenced by the fine structure of the islands. For large islands and field-line pitch, a carbon radiation belt is formed on the inboard side of the torus and is kept outside the LCFS, leaving a warm region for the recycling neutrals. Figure 16 shows the typical location of the carbon radiation and the distribution of the hydrogen ionization source including molecule contributions. They are code results for the standard divertor configuration for which stable partial detachment is achieved in experiments. The low-field side and the divertor recycling region are relatively radiation-free so that the power fraction entering the islands on the low-field side can be transported down to the divertor region to sustain the recycling process. The existence of such a power channel has been detected by target thermography, as shown by the right lower picture in figure 16 . The power channel leaves a hot spot at its termination on a target, which agrees well with the code results (see the right upper picture in figure 16). This is the reason why the detachment in W7-AS is termed 'partial detachment'.

\subsection{Marfe-like phenomena}

In contrast to the inboard-side-radiation picture for a stable partial detachment configuration, the EMC3/EIRENE 


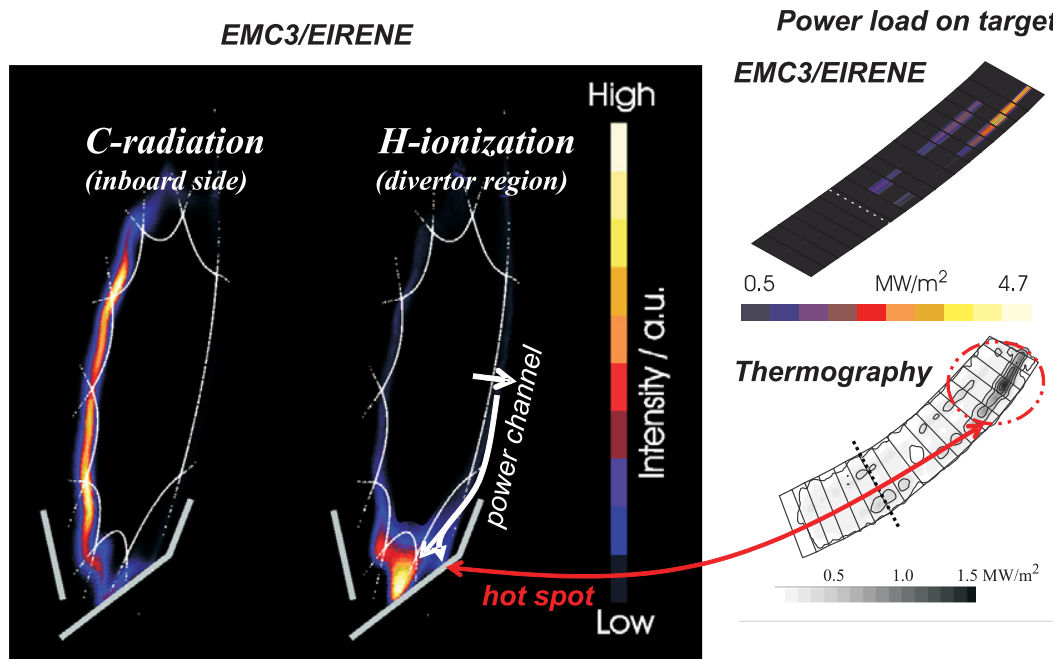

Figure 16. Typical distribution patterns of carbon radiation and hydrogen ionization in a stable partial detachment for the W7-AS standard divertor configuration. A radiation-free power channel on the low-field side transporting energy down to the recycling region is detected by the target surface thermography, in good agreement with the code results.
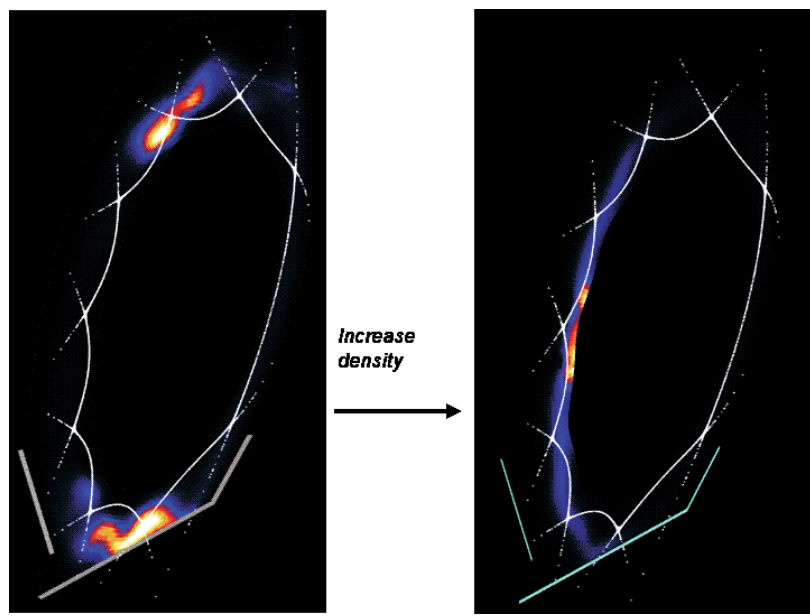

Figure 17. EMC3/EIRENE shows that a small island size or field-line pitch results in an intensive, divertor-localized radiation (left) in the initial detachment phase. However, independent of the island configurations, once plasma approaches a density limit, a strongly localized radiation zone appears on inboard midplane (right).

predicts an intensive, Marfe-like radiation distribution for configurations with small islands or small field-line pitch in the initial detachment phase before the radiation layer moves into the core with increasing density. The left picture in figure 17 shows the carbon radiation distribution for an island configuration in which the target-to-core distance is reduced by roughly a factor of 2 with respect to the case shown in figure 16. Now, the radiation layer stays in the divertor region and cools down the recycling zone. The islands become more transparent for the recycling neutrals. The EIRENE code shows that, in this case, the neutral penetration flux into the core is much more sensitive to the SOL power and the separatrix density than in the inboard side radiation case. A simple stability analysis shows that the penetrating neutrals can drive an instability [10]. The loss of the island neutral screening is considered to be the reason for the detachment instability observed in experiments with such a configuration. However, independent of the island configuration, the 3D code shows that the radiation layer always moves towards the inboard midplane when the plasma approaches a density limit (see the right picture in figure 17). In this case, the radiation layer is located inside the LCFS which has been observed by a CCD-camera viewing a whole low divertor plate [30,31]. The radiation on closed flux surfaces is associated with a thermal, Marfe-like instability and always leads to a strong degradation of the energy confinement.

\section{Discussion and conclusion}

The divertor-relevant field-line pitch in an island divertor arises from the shear, rather than from the rotational transform itself, as is the case in the tokamak poloidal-field divertor. This explains why the island divertors usually have much larger connection lengths than tokamak divertors in spite of the smaller target-to-core distances limited by the finite radial island size. As a consequence, the cross-field transport generally plays a more important role in the island divertor than in a tokamak divertor. Because the cross-field transport is strongly associated with the specific island geometry, a proper assessment of the island divertor transport needs a 3D code.

The tokamak two-point model has been extended to cover the island divertor by including cross-field transport contributions. The used approach is strongly influenced by the 3D modelling experience and results gathered from W7AS and mainly aims at explaining the governing physics and mechanisms behind the $3 \mathrm{D}$ simulation findings and experimental observations. The divertor-relevant field-line pitch in W7-AS is by two orders of magnitude smaller than that in tokamaks. Associated with the small islands and the discontinuous targets, cross-field particle diffusion and viscous transport lead to damping of the parallel counterflows on different parts of the islands. This geometryrelated momentum loss breaks up the linear pressure coupling between upstream and downstream already at low densities, high temperatures. A high-recycling regime based on parallel 
pressure conservation therefore does not appear in W7AS, which is predicted by the $3 \mathrm{D}$ code and confirmed by experiments. Although most recycling neutrals are stopped in the island SOL, the recycling neutral flux penetrating into the core still provides the main fuelling source for the core even in the presence of a strong NBI source. Thus, the core plasma is strongly coupled with the SOL plasma through the recycling neutral gas. Both the experimentally observed abrupt change in the separatrix density during a density scan at the transition from $\mathrm{NC}$ to $\mathrm{HDH}$ plasma conditions and the experimentally observed geometry-related detachment instability can be explained by the island neutral screening effect. In a moderate island density range where the island temperature is still higher than the ionization energy of the working gas, the island neutral screening is efficient in the sense that the core fuelling rate from the recycling neutral gas is saturated or even decreases with an increase in the separatrix density. In order to further increase the core density, the island screening has to be overcome by cooling down the islands. This is consistent with the experimental observation that strong gas puffing is necessary in order to access the $\mathrm{HDH}$ plasmas. The HDH plasma is surrounded by a cold, dense island plasma of several centimetres thickness. Numerical studies using the EMC3/EIRENE code show that, under HDHconditions, the edge islands have a strong retention effect on the intrinsically-released impurities. Under this condition, the cross-field heat conduction, due to the small field-line pitch in W7-AS, strongly reduces the parallel heat conductive flux, especially for ions, and thereby reduces the related thermal forces. A purely friction-dominated SOL regime can be established under HDH-plasma conditions, in which the frictional plasma flow drags the impurity back to the targets. The carbon separatrix density, which is normalized to the total production rate in order to isolate the transport effect from the production processes, is reduced by more than one order of magnitude with respect to the thermal-force-dominated case typical for low densities. The transition from thermal-forcedominated to friction-dominated impurity transport is sharp because of the high sensitivity of the classical heat conductivity to the temperature.

When the plasma density, starting from the HDH-regime, is increased further, the plasma detaches in the sense that carbon radiation jumps to a high radiation level ( $>80 \%$ of $P_{\mathrm{SOL}}$ in simulations and $>90 \%$ in experiments) and the radiation layer lifts from the targets towards the inner separatrix. Detachment for a plasma heated by $2 \mathrm{MW}$ is achieved in experiments at a line-averaged density of $3 \times 10^{20} \mathrm{~m}^{-3}$ and an $n_{\mathrm{es}}$ of $6 \times 10^{19} \mathrm{~m}^{-3}$ ! The absence of a high-recycling regime accounts for this high-density condition for detachment transition, which is predicted by the $3 \mathrm{D}$ code and can be explained by the simple extended two-point model. The abrupt change in radiation level and position during transition can be well understood by analysing the radial power balance including the radiation term. However, a more precise localization of the radiation layer in a detached plasma needs the EMC3/EIRENE code. It is found that the fine island structure strongly affects the carbon radiation distribution, which can influence the stability of a detached plasma. For large islands with sufficiently large field-line pitch, for which stable partial detachment is achieved in experiments, the 3D code shows an inboard-side located radiation layer outside the LCFS. This is supported by target surface thermography measurement. In contrast, a small island or field-line pitch results in an intensive, X-point-Marfe-like radiation in the recycling zone. The loss of neutral screening in this case is considered to be the reason for the detachment instability observed in experiments. When the plasma density approaches a density limit, the radiation layer goes finally into the core and moves to the inboard midplane, independent of the island configurations. An unstable, Marfe-like radiation originating at the inboard midplane is detected by a CCD-camera for deeply detached plasmas.

\section{References}

[1] Grigull P. et al 2001 Plasma Phys. Control. Fusion 43 A175

[2] Ohyabu N. et al 1994 Nucl. Fusion 34387

[3] Morisaki T. et al 2005 J. Nucl. Mater. 337-339 15

[4] König R. et al 2002 Plasma Phys. Control. Fusion 442365

[5] McCormick K. et al 2002 Phys. Rev. Lett. 89015001

[6] Burhenn R. et al 2004 Fusion Sci. Technol. 46115

[7] Renner H. et al 2000 Nucl. Fusion $\mathbf{4 0} 1083$

[8] Feng Y. et al 1999 J. Nucl. Mater. 266-269 812

[9] Reiter D. 1984 Technical Report Jül-1947, KFA Jülich, Germany

[10] Feng Y. et al 2005 Nucl. Fusion 4589

[11] Feng Y. et al 2002 Plasma Phys. Control. Fusion 44611

[12] Mahdavi A.M. et al 1981 Phys. Rev. Lett. 471602

[13] Borras K. 1991 Nucl. Fusion 311035

[14] Stangeby P.C. 1993 Nucl. Fusion 331695

[15] Pitcher C.S. and Stangeby P.C. 1997 Plasma Phys. Control. Fusion 39779

[16] Feng Y., Kisslinger J. and Sardei F. 1999 26th EPS Conf. on Controlled Fusion and Plasma Physics (Maastricht) vol 23 (ECA) p 1465

[17] Grigull P. 2006 private communication, Max-Planck-Institut fuer Plasmaphysik, 17491 Greifswald, Germany

[18] Lehnert B. 1983 Nucl. Fusion 231327

[19] Stangeby P.C. et al 1995 Nucl. Fusion 351391

[20] Feng Y. et al 2005 32nd EPS Conf. on Controlled Fusion and Plasma Physics (Tarragona) vol 29C (ECA) P 1.012

[21] McCormick K. et al 2003 J. Nucl. Mater. 313-316 1131

[22] Igitkhanov Y. 1988 Contrib. Plasma Phys. 28477

[23] Grigull P. et al 2003 Fusion Eng. Des. 66-68 49

[24] Janeschitz G. et al 1992 J. Nucl. Mater. 196-198 380

[25] Petrie T.W. et al 1992 J. Nucl. Mater. 196-198 848

[26] Borras K. and Janeschitz G. 1994 Nucl. Fusion 341203

[27] Baker D.R. et al 1982 Nucl. Fusion 22807

[28] Lipschultz B. et al 1984 Nucl. Fusion 24977

[29] McCormick K. et al 2001 28th EPS Conf. on Controlled Fusion and Plasma Physics (Madeira) vol 25A (ECA) p 2097

[30] Thomsen H. et al 2004 Nucl Fusion. 44820

[31] Wenzel U. et al 2002 Plasma Phys. Control. Fusion 44 L57 